\title{
The directions of the development of the methods for designing the geometric form of mechanical structures
}

\author{
Andrzej Kołodziej ${ }^{1,},{ }^{*}$, Marian Dudziak $^{1}$, and Krzysztof Talaśka ${ }^{2}$ \\ ${ }^{1}$ The President Stanisław Wojciechowski State University of Applied Sciences in Kalisz, Kalisz, \\ 62-800 Poland \\ ${ }^{2}$ Chair of Basics of Machine Design, Poznan University of Technology, Poznan, 60-965, Poland
}

\begin{abstract}
In the paper the main directions of the development of the methods for designing selected geometrical features of the mechanical components along with its representation in technical drawing were described. The influence of the shape deviations on the condition of the mechanical connections and its fits was defined and characterized. It was proved, that the shape obtained in the machining process significantly differs from the nominal shape of the designed parts. The consequence of the following deviations are the troubles during cooperation of the parts, which perform either rotary or reciprocating motion. The results presented in this paper contain the measurement of the actual geometry of the journal and the hub along with determination of the value and the type of their shape deviations. Authors have proved that the magnitude of the shape deviations are subject to the errors connected with the methods of measurement and used tools. Proper simulations were performed, which shows the certain influence of the shape deviations on the cooperation of the mechanical parts.
\end{abstract}

\section{Introduction}

The physical and geometric form of the mechanical structure components decides the functional integration of the final assemblies and subassemblies. This has a material influence on the durability, reliability and safety of the construction $[1,2]$.

The properties and features of the construction are affected by the macrogeometry of the surface of integrated components, and in particular any deviations of shape (linearity, roundness and cylindricity) $[1,2]$. The values of these deviations affect, among others, the existing area of contact of integrated items within a matching pair of components, which in many cases causes a reduction of the assumed durability and tribological criteria. It is therefore relevant to select and design proper fitting and shape tolerance of components, the axial alignment of integrated components, ensuring rigidity and global durability in the

\footnotetext{
${ }^{*}$ Corresponding author: a.kolodziej@pwsz.kalisz.pl

Reviewers: Grzegorz Domek, Radostaw Pytliński
} 
place of actual contact, together with susceptibility to vibration and energy dissipation. Hence, there is a recognized need for a methodological approach to design and evaluation of coupling surfaces used in different normalized and non-normalized structural machine components.

In order for the decisions formulated at the stage of selecting construction solutions used for the connections to be optimal, it is required to specify the allowed area of shape deviation, area and dimensions of integrated components in comparison to the nominal values specified in the documentation during the preliminary design stage. The optimal selection and proper representation of the desired shape tolerance in the construction documentation is ensured by state of the art knowledge, the ability analyze as well as its correct interpretation. This design stage should call for the knowledge of methods of manufacturing, the engineering processes of taking measurements as well as the evaluation of the product, installation and operation. Fulfilling the conditions for this task is facilitated by the knowledge of manufacturing engineering, e.g. the machine park, special tools, milling machines as well as the geometric properties of the machined item, as they are the main causes for deviations during forming of the surfaces [1,2].

Each subsequent stage of product manufacturing - starting with the design stage and establishing product specification on the construction drawing, through manufacturing (interpretation of the specification), verification using selected measuring instrumentation (measurement consistency) and final assembly - can be burdened with certain incorrections which aggregate during the entire development process. Therefore, the knowledge of objective design representation is required. The ambiguity present during product development caused by the current representation of shape tolerance of axisymmetric connections and their effect on the operation under conditions of both static and dynamic loads, is demonstrated in the example of the manufacturing of a hub and its integrated journal (orifice and a shaft). The representation of the construction as provided in the technical documentation is prepared by the ordering entity, whereas the components of the connection: the hub and the shaft, are manufactured in collaboration by different plants. Afterwards, they are joined together to form a complete assembly by the ordering entity $[1,2]$.

\section{The philosophy of design and manufacturing}

The design process mostly entails selecting the necessary construction features: the state of geometric form, the state of surface geometry, material, static, dynamic and fatigue strength, as well as the legal properties allowing to best meet the functional, operational and safety requirements.

Axisymmetric connections are among the most widespread in machine construction. Proper design methodology of the components is very important for manufacturing durable and effective machine assemblies. The correct and lasting operation of the connection comprising a hub and a shaft is affected by the quality of workmanship of individual components based on the design representation, in particular with regards to the methodology of measuring the actual shape in relation to the deviations in the longitudinal and transverse cross sections of the connection. Fig. 1 demonstrates model shape deviations in longitudinal and transverse cross-section. Whereas Fig. 2 presents an assembly view of shape deviation along the transverse cross-section (triangular lobing) and along the longitudinal cross-section (saddleback distortion). Proper identification of the shape distortion, both along the transverse and longitudinal cross-section, together with its dimensions affect the further process of selecting the components to form the designed connection. 
a)
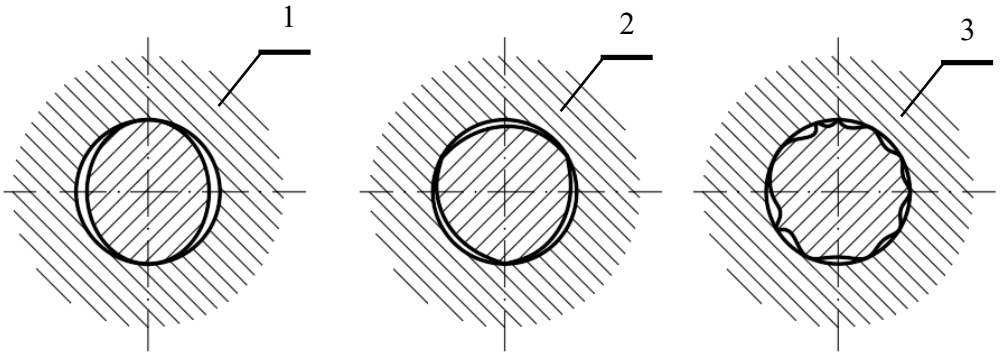

b)
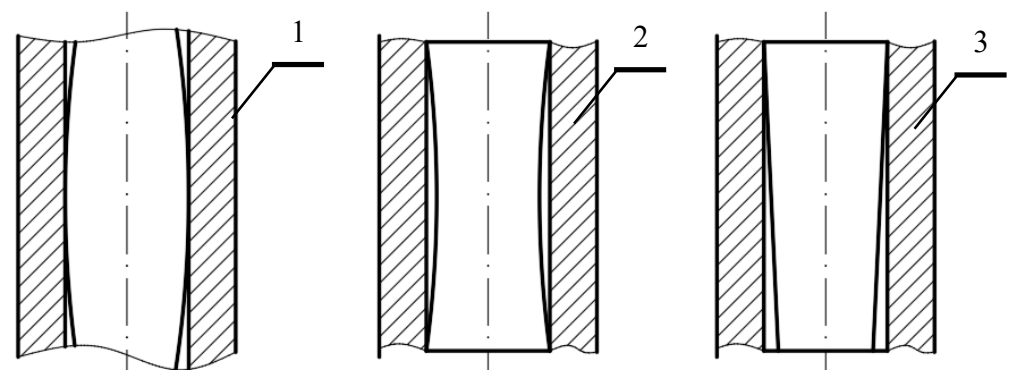

Fig. 1. Shape deviation models: a) in transverse cross-section 1 - oval distortion, 2 - triangular lobing, 3 - multi-angular lobing; b) in longitudinal cross-section: 1 - barrel-shape distortion, 2 - saddleback distortion, 3 - conical distortion

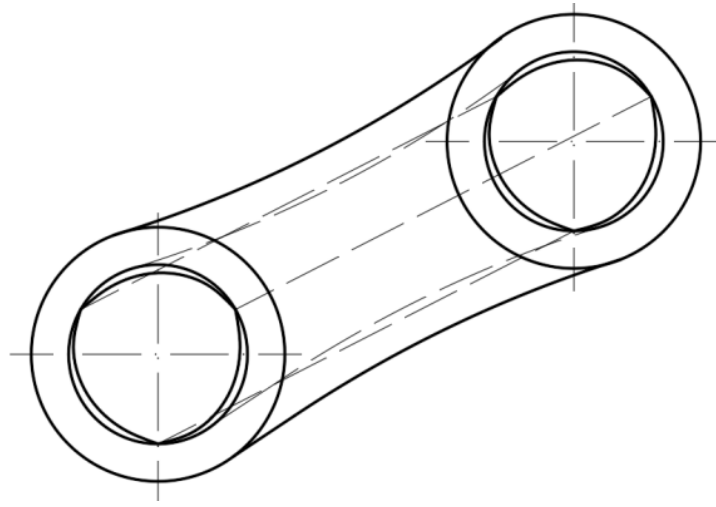

Fig. 2. Assembly view of shape distortion along transverse cross-section (triangular lobing) and transverse cross-section (saddleback distortion)

\section{The aim of this work}

There is a recognized need to improve the methods of dimensioning and specification of tolerance to include the measurement methodology as well as define the particular actions thereof. This allows to avoid subjective and often inadequately thought out determinations which affect the identification of objective numerical values of measured geometric characteristics affecting the product quality.

The principles of specification of shape tolerance that are presently in use along with the representation on drawing documentation does not provide sufficient information and guidelines for taking the measurements and evaluating the results.

It is furthermore not possible to make uniform arrangements regarding:

- measuring strategy,

- type of replacement component,

- filter characteristic, 
- geometry and radius of the measuring tip,

- measuring force and speed.

In industrial practice, the measurement results are sometimes manipulated if the desired value of geometric deviation is not satisfactory (exceeds the assumed tolerance value). The parameters are altered and chosen so that the end results of measurement produce deviation values within the specified tolerance. This means that the aim is for the observed surface (surface profile) to be within tolerance range.

When determining the roundness deviation, the actual outline of any cross-section of the cylindrical surface within tolerance value should not fall outside the area of two concentric circles on a single plane with the difference of radii equal to the tolerance value (Fig. 3). In the example under consideration $T=0.03 \mathrm{~mm}$. This is known by the operator of the measuring instrumentation attempting to measure the roundness deviation.

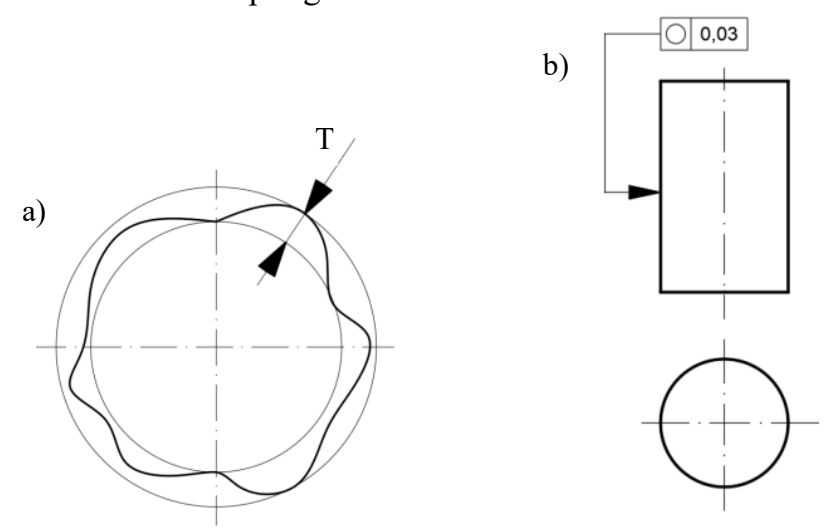

Fig. 3. Roundness tolerance: a) definition, b) example

Whereas for the measurement to be performed in a manner that is metrologically correct and the result to be reliable, it is required to unambiguously specify its following conditions:

- measurement strategy (number of points of measurement, distance of the plane of the measured outlined of roundness from the edge of the area of observation),

- metrological characteristics of the measuring tip (geometry and radius, measurement force, measurement speed),

- filter characteristics and filtering conditions,

- used reference element (MZC, MCC, MIC, LSC).

\section{Methodology and scope of measurement examination}

An attempt to identify selected ambiguities of measurement of roundness resulting from different conditions of its performance was made on a coordinate based measurement probe system Contura G2 by Carl Zeiss.

The examination tasks were performed on 30 pieces of shafts manufactured by the milling industry, randomly selected from a production batch (500 pcs.), with nominal diameter $\varnothing=56.38 \mathrm{~mm}$ and length $L=92.04 \mathrm{~mm}$. During the measurement of roundness deviation from the diameter value $\varnothing=56.38 \mathrm{~mm}$ only one of the parameters were changed in sequence, while other parameters remained constant. The following parameters were changed:

- measuring method and number of points of measurement (impulse measurement 4, 816,32 and 64 points; scanning measurement - 3203 points),

- reference elements (Chebyshev's, Escribed, Inscribed, Gaussian), 
- the distance of the plane of the measured roundness outline from the lower head of the shaft (23 mm, $46 \mathrm{~mm}, 69 \mathrm{~mm})$,

- filter characteristics (50 UPR, $150 \mathrm{UPR}, 500 \mathrm{UPR}$ ),

- measuring tip radius $(1.75091 \mathrm{~mm}, 3.50025 \mathrm{~mm}, 4.00012 \mathrm{~mm})$,

- measurement speed $(20 \mathrm{~mm} / \mathrm{s}, 50 \mathrm{~mm} / \mathrm{s}, 150 \mathrm{~mm} / \mathrm{s})$,

- measurement force $(100 \mathrm{mN}, 200 \mathrm{mN}, 400 \mathrm{mN})$.

The first measurements were performed using the impulse method for 4, 8, 16, 32 and 64 points of measurement as well as a scanning measurement with 3203 points of measurement (Fig. 4). The operator does not know the model for roundness deviation when placing the item in the measuring area of the machine. Too low number of points of measurement may cause extreme points of the silhouette to be omitted. Scanning measurement allows to achieve a large number of points of measurement, which provides significant information regarding the tested item. In this regard, the analysis is similar to measurements carried out on specialized equipment. However, due to the significant costs involved, a large number of machines are only fitted with impulse measurement heads. Additionally, the impulse measurement is much more accurate than scanning measurement. However, one needs to consider if the limited number of points of measurement does not cause too much ambiguity in measurement, which are particularly noticeable with a small number of measurement points. Figs. 5-10 present the values of measured deviation with variance of the specified parameter, which has been indicated in measurement parameters for each case.

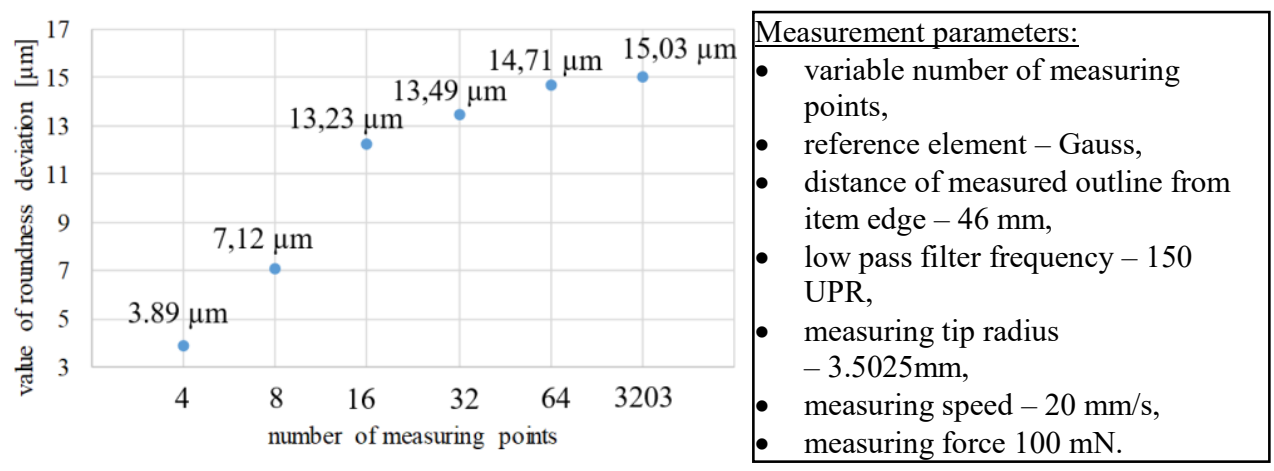

Fig. 4. Values of roundness deviations obtained by pulse method for a variable number of measuring points: $4 \mathrm{p}-3.89 \mu \mathrm{m}, 8 \mathrm{p}-7.12 \mu \mathrm{m}, 16 \mathrm{p}-13.23 \mu \mathrm{m}, 32 \mathrm{p}-13.49 \mu \mathrm{m}, 64 \mathrm{p}-14.71 \mu \mathrm{m}, 3203 \mathrm{p}-$ $15.03 \mu \mathrm{m}$

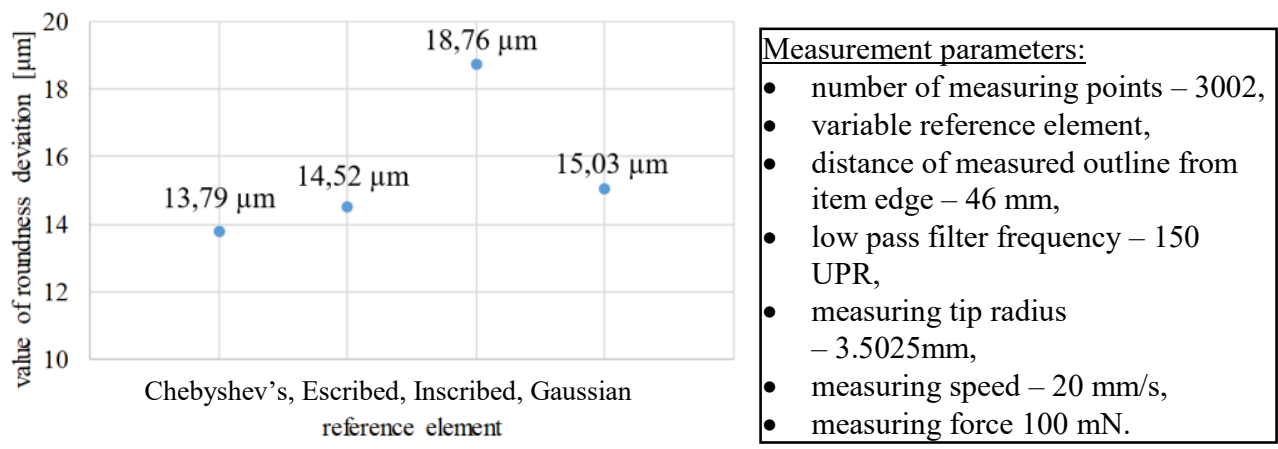

Fig. 5. Roundness deviations values obtained using different reference elements: Chebyshev -13.79 $\mu \mathrm{m}$, Escribed $-14.52 \mu \mathrm{m}$, Inscribed $-18.76 \mu \mathrm{m}$, Gauss $-15.03 \mu \mathrm{m}$ 


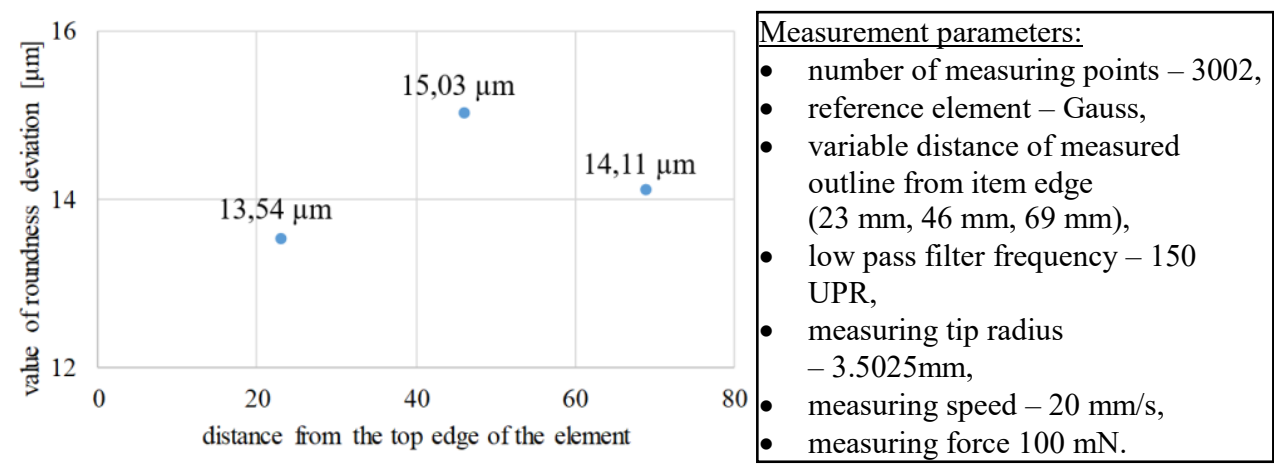

Fig. 6. Values of roundness deviations obtained during the measurement at different heights of the journal: $23 \mathrm{~mm}-13.54 \mu \mathrm{m}, 46 \mathrm{~mm}-15.03 \mu \mathrm{m}, 69 \mathrm{~mm}-14.11 \mu \mathrm{m}$
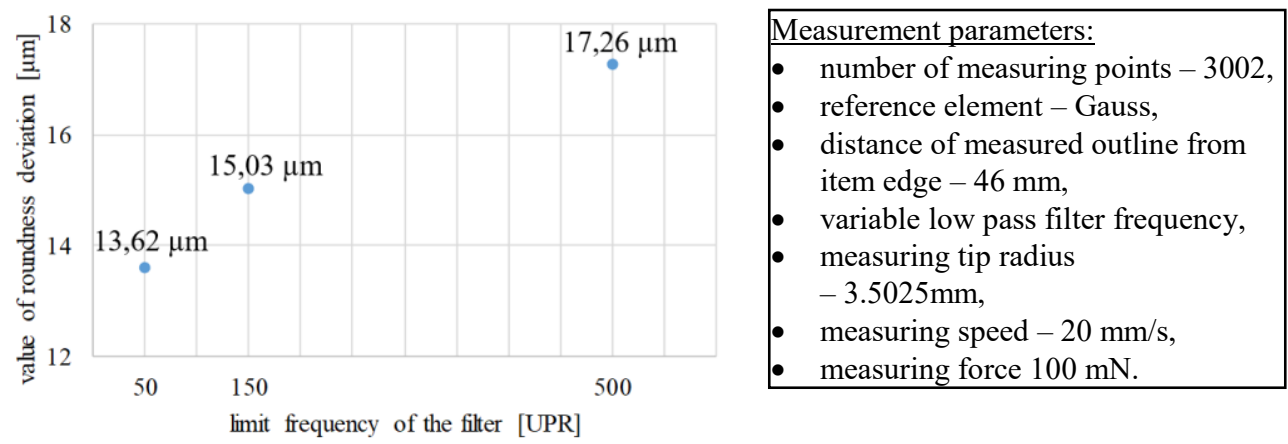

Fig. 7. Values of roundness deviations obtained using low-pass filters with different limit frequencies: $50 \mathrm{UPR}-13.62 \mu \mathrm{m}, 150 \mathrm{UPR}-15.03 \mu \mathrm{m}, 500 \mathrm{UPR}-17.26 \mu \mathrm{m}$
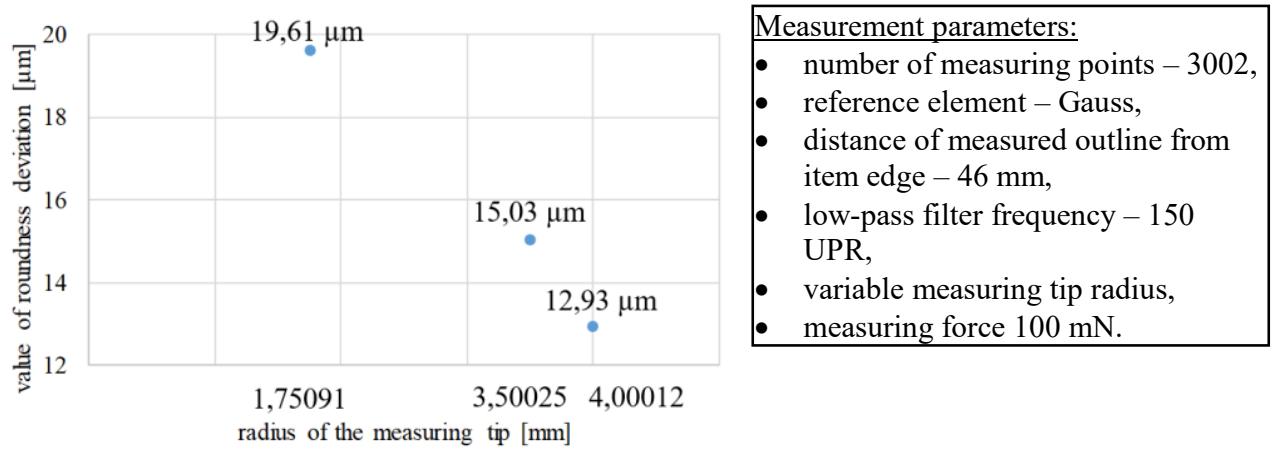

Fig. 8. Values of roundness deviation obtained using different radii of measuring tips: $r=1.75091$ $\mathrm{mm}-19.61 \mu \mathrm{m}, \mathrm{r}=3.50025 \mathrm{~mm}-15.03 \mu \mathrm{m}, r=4.00012 \mathrm{~mm}-12.93 \mu \mathrm{m}$ 


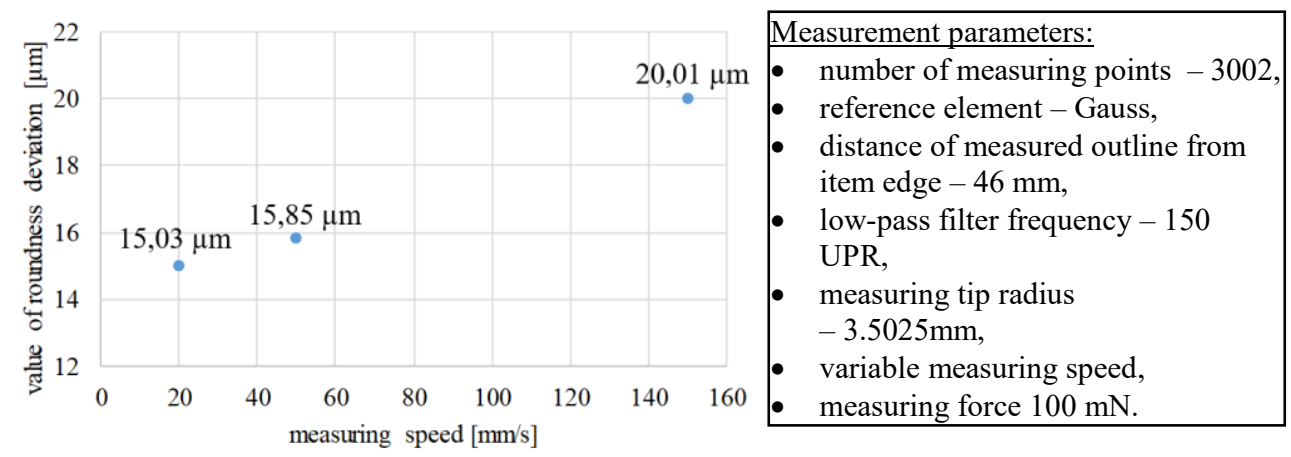

Fig. 9. Values of roundness deviations obtained at different measurement speeds: $20 \mathrm{~mm} / \mathrm{s}-15.03$ $\mu \mathrm{m}, 50 \mathrm{~mm} / \mathrm{s}-15.85 \mu \mathrm{m}, 150 \mathrm{~mm} / \mathrm{s}-20.01 \mu \mathrm{m}$

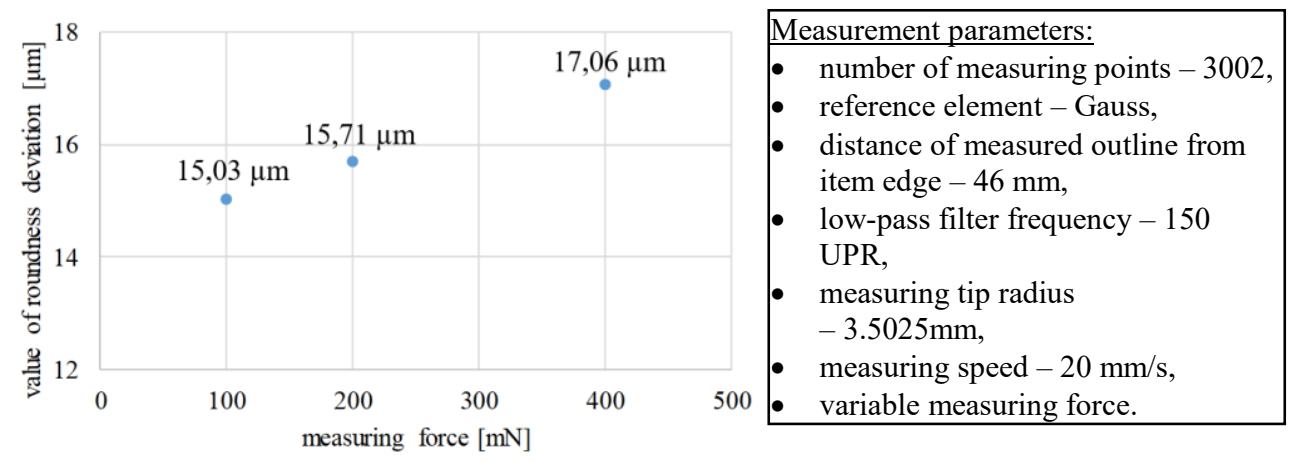

Fig. 10. Values of roundness deviations obtained at various measuring forces: $100 \mathrm{mN}-15.03 \mu \mathrm{m}$, $200 \mathrm{mN}-15.71 \mu \mathrm{m}, 400 \mathrm{mN}-17.06 \mu \mathrm{m}$

\section{The influence of the type of deviation and its value on the functional parameters of the axisymmetric connection}

Works [3-7] present detailed results of simulation studies of the influence of the type of deviation and its value on the functional parameters of the axisymmetric connection. These studies demonstrate the advantage of multi-angular lobing and upper grades of workmanship accuracy on the decrease of simplified stress value in the contact zone of the connection. Additionally, it was confirmed that such configurations of the actual shape of components reduce the parameters defining the integration with regards to dynamic loads. Figs. 11 and 12 present the change in of the frictional torque and frictional force required to dismantle a connection formed with a 13-angle shaft with diameter $19 \mathrm{~mm}$ with manufacturing accuracy classes of IT4, IT6 and IT9 as a function of the angular position of the journal relative to the hub. Only the gravitational load is affected on the connection. Analyzing the results provided in work [7], we observe an unambiguous influence of the rotational speed of the connection components on the anchoring reaction value, its value being additionally increased by higher tolerance values of the manufactured components. 


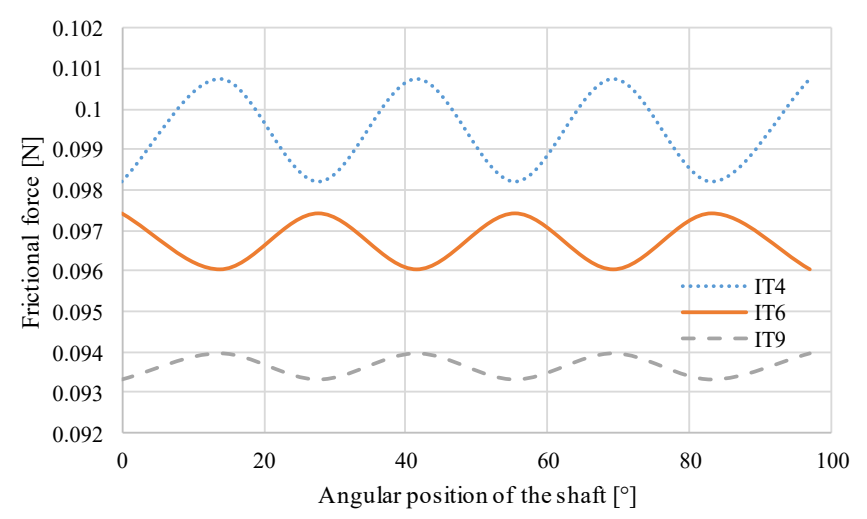

Fig. 11. Change of frictional force as a function of the angular position of the journal in relation to the hub [6]

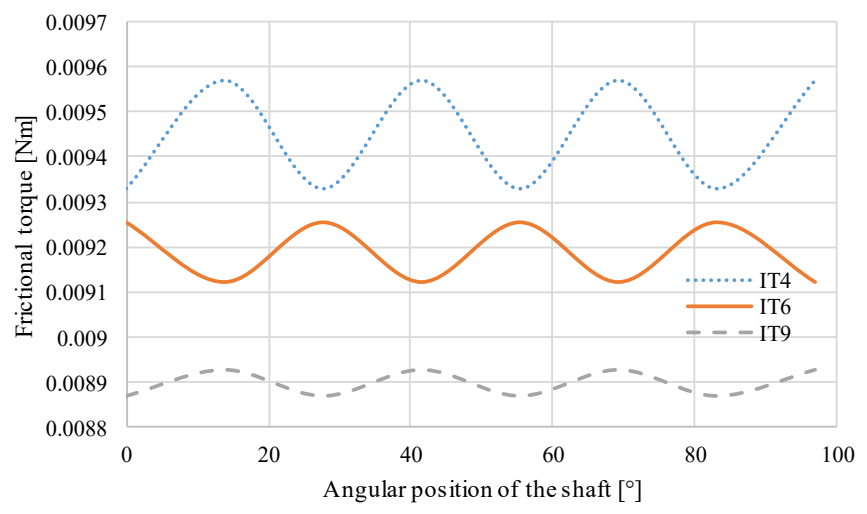

Fig. 12. Change of the frictional torque as a function of the angular position of the journal in relation to the hub [6]

\section{Conclusion}

Analyzing the results of studies presented in this work together with the studies of works [3-7] the following conclusions may be drawn:

- shape deviations significantly limit the area of contact between the components of the connection,

- the most useful methodologies for the evaluation of the outlines of cylindrical items are the grid analysis and measuring the outline roundness,

- frictional resistance during the sliding and turning of the journal (during use) depend on the angular position in the orifice,

- the model of deviation of the outline shape is decisive for the hub anchoring reaction during the turn of the journal.

For the sake of further study of this issue, it is called for to perform studies for different types of shape deviation, tolerance values and nominal dimensions. The range of experiments to account for the dynamic loads in an assembly calls for using additional loads affecting the connection. 


\section{References}

1. S. Adamczak, Geometrical measurements of surface - shape, waviness and roughness. WNT, Warsaw (2008)

2. S. Adamczak, W. Makieła, K. Stępień, Investigating advantages and disadvantages of the analysis of a geometrical surface structure with the use of Fourier and wavelet transform, Metrology and Measurement Systems, XVII, pp. 233-244 (2010)

3. M. Dudziak, G. Domek, A. Kołodziej, K. Talaśka, Contact Problems Between the Hub and the Shaft with a Three-angular Shape of Crosssection for Different Angular Positions, Procedia Engineering, 96, pp.50-58 (2014)

4. M. Dudziak, A. Kabała, A. Kołodziej, The Topic of Contact Zone Problems in the Shaft and Hole Joint, Taking into Account Component Form Errors, Journal of Mechanics Engineering and Automation, 3, pp. 586-590 (2013)

5. M. Dudziak, G. Domek, A. Kołodziej, K. Talaśka, Contact Problems Between the Hub and the Shaft with a Four-Angular Shape of CrossSection for Different Angular Positions, Applied Mechanics and Mechatronics II (Applied Mechanics and Materials), pp. 54-62 (2015)

6. M. Dudziak, G. Domek, A. Kołodziej, K. Talaśka, Multi-angularity - identification of parameters and compatibility conditions of the axisymmetric connection with form deviations, Procedia Engineering, 177, pp. 431-438 ( 2017 )

7. M. Dudziak, A. Kołodziej, K. Talaśka, Cooperation of axisymmetric connection elements under dynamic load, MATEC Web of Conferences, 157, 02017 (2018) 